\title{
Tratamiento del síndrome de intestino irritable por gastroenterólogos: resultados de un estudio de corte transversal a partir de una encuesta elaborada por expertos
}

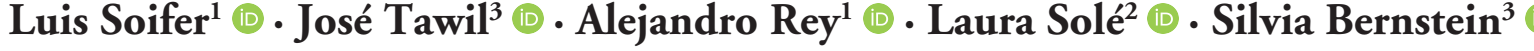 \\ ${ }^{1}$ Instituto CEMIC. Ciudad Autónoma de Buenos Aires, Argentina. \\ ${ }^{2}$ Consultorios de Motilidad Digestiva (CMD). \\ ${ }^{3}$ Gastroenterología Diagnóstica y Terapéutica GEDyT.
}

Acta Gastroenterol Latinoam 2021;51(3):280-291

Recibido: 19/01/2021 / Aceptado: 30/07/2021 / Publicado online: 27/09/2021 / https://doi.org/10.52787/kksx5531

\section{Resumen}

Introducción. El sindrome de intestino irritable (SII) ocasiona un deterioro importante en la calidad de vida de quienes lo padecen y es un motivo común de consulta en gastroenterología. El tratamiento de este sindrome incluye una amplia variedad de fármacos y de recomendaciones basadas en diversos factores de su fisiopatología. Sin embargo, los resultados suelen ser variables y en ocasiones no resultan satisfactorios ni para el paciente ni para el médico tratante. El objetivo principal del presente trabajo es describir las terapéuticas que emplean los gastroenterólogos en el manejo del SII en la Argentina. Materiales y métodos. Cuestionario de cuarenta y siete preguntas, elaborado por expertos, difundido mediante las entidades societarias representantes de la especialidad en la Argentina y respondido por los especialistas vía Google Forms. Resultados. 304 respuestas,

Correspondencia: Alejandro Rey

Correo electrónico: doctoralejandrorey@gmail.com
157 de mujeres (51,6\%) y 147 de hombres (48,4\%). Edad media de 44 y 49,9 años respectivamente. El 87,5\% considera como objetivo principal la mejoría en la calidad de vida. Con respecto a la fisiopatología del sindrome de intestino irritable variante diarrea (SII-D), el 49,5\% lo atribuye a estrés emocional, mientras que para el sindrome de intestino irritable variante constipación (SII/C), un 42,8\% lo considera multifactorial, con predominio del tránsito colónico lento. Ninguna opción de tratamiento fue valorada como muy efectiva por más del 50\% de los encuestados. La rifaximina fue considerada el tratamiento más efectivo para el SII-D, en tanto que el polietilenglicol lo fue para el SII-C. Se observaron entre los subespecialistas diferencias estadisticamente significativas en el tratamiento del SII-D respecto de la efectividad considerada para la dieta, la amitriptilina y los antibióticos distintos de la rifaximina, y respecto de la efectividad considerada para el bisacodilo/picosulfato y los antibióticos en el manejo del SII-C. Conclusión. El mecanismo fisiopatológico considerado más frecuente en el SII-D es el estrés. La gran mayoría de los encuestados considera que la meta principal del tratamiento es la mejoría de la calidad de vida, por sobre el alivio sintomático. Ninguna de las drogas es considerada muy efectiva en el tratamiento de las distintas variantes del SII por al menos el 50\% de los encuestados. Se observaron diferencias estadisticamente significativas en la proporción de consultas mensuales por SII en relación con la edad y en la efectividad considerada para algunos tratamientos en relación con la subespecialidad de los médicos 
encuestados. El abordaje terapéutico del SII por los gastroenterólogos argentinos es variado y algo heterogéneo, lo cual resulta en un manejo todavía inapropiado de esta condición.

Palabras claves. Sindrome de intestino irritable, trastornos funcionales colónicos, diarrea, estreñimiento, dolor abdominal.

\section{Irritable Bowel Syndrome Treatment by Gastroenterologists: Results of a Cross-Sectional Study from an Expert Designed Survey}

\section{Summary}

Introduction. Irritable bowel syndrome (IBS) accounts for a significant deterioration in the quality of life of patients who suffer from it and is a common reason for consultation in gastroenterology. The treatment of this syndrome includes a wide variety of medications and recommendations based on different factors of its physiopathology. However, the results are usually variable and sometimes unsatisfactory for either the patient or the treating physician. The main objective of the present work is to describe the different treatments used by gastroenterologists in the management of IBS in Argentina. Materials and methods. Questionnaire of forty-seven questions, prepared by experts, distributed by medical associations of gastroenterology in Argentina and answered by specialists via Google Forms. Results. 304 responses, 157 from women (51.6\%) and 147 from men (48.4\%). The mean age was 44 and 49.9 years, respectively. 87.5\% consider improvement of quality of life as the main goal of treatment. Regarding physiopathology of diarrhea variant irritable bowel syndrome (IBS-D), 49.5\% considered emotional stress, while for constipation variant irritable bowel syndrome (IBS-C), 42.8\% considered it multifactorial, with a predominance of slow colonic transit. No treatment option was rated as very effective by more than 50\% of the respondents. Rifaximin was considered the most effective treatment in IBS-D, while polyethylene glycol was for IBS-C. Statistical significant differences were observed among gastroenterology subspecialties in the treatment of IBS-D with respect to the efficacy of diet, amitriptyline and antibiotics other than rifaximin, and regarding the effectiveness for bisacodyll picosulfate and antibiotics in the treatment of IBS-C. Conclusion. The most common pathophysiological mechanism in IBS-D is stress. The vast majority of respondents consider that the main goal of treatment is the improvement of the quality of life, over symptomatic relief. None of the drugs is considered very effective in the treatment of the different IBS variants by at least $50 \%$ of those surveyed. Statistical- ly significant differences were observed in the proportion of monthly consultations for IBS regarding to age and in the effectiveness considered for some treatments in relation to the subspecialty of physicians. The therapeutic approach to IBS by Argentine gastroenterologists is varied and somewhat heterogeneous, resulting in a still inappropriate management of this condition.

Keywords. Irritable bowel syndrome, colonic functional disorders, diarrhea, constipation, abdominal pain.

\section{Abreviaturas}

SII: Sindrome de intestino irritable.

SII-D: Sindrome de intestino irritable variante diarrea.

SII-C: Sindrome de intestino irritable variante constipación. IBS: Irritable bowel syndrome.

IBS-D: Diarrhea predominant irritable bowel syndrome.

IBS-C: Constipation predominant irritable bowel syndrome.

FAGE: Federación Argentina de Gastroenterología.

SAGE: Sociedad Argentina de Gastroenterología.

CIDEMO: Círculo de la Motilidad del Aparato Digestivo.

AMBA: Area Metropolitana de Buenos Aires.

OR: Odds ratio.

IC 95: Intervalo de confianza 95\%.

PEG: Polietilenglicol.

\section{Introducción}

Los trastornos funcionales digestivos, y en particular el SII, son condiciones muy prevalentes, que ocasionan un deterioro importante en la calidad de vida y son un motivo de consulta frecuente, tanto en la atención primaria como en las consultas especializadas. Los pacientes buscan un diagnóstico, muchas veces difícil de precisar, y un tratamiento eficaz que los ayude a paliar los síntomas. ${ }^{1,2}$

De acuerdo con los criterios de Roma IV, el SII se diagnostica por la presencia de dolor abdominal recidivante, que ha de estar presente al menos un día a la semana y con dos o más de las siguientes características: se asocia a la defecación; está relacionado con un cambio en la frecuencia de las deposiciones; se asocia con un cambio en la consistencia de las deposiciones. En cuanto al marco temporal, estos criterios deben cumplirse durante los últimos tres meses y los síntomas deben haber comenzado como mínimo seis meses antes del diagnóstico. ${ }^{3}$

Tradicionalmente, el enfoque terapéutico del SII se ha basado en la sintomatología referida por el paciente: tratar el dolor, la diarrea o la constipación. En 
la actualidad, sin embargo, se ha producido un cambio paradigmático, que busca orientar el tratamiento sobre la base del conocimiento de los posibles mecanismos fisiopatológicos involucrados. En este sentido, las diferentes terapéuticas se enfocan, por ejemplo, en la hipersensibilidad visceral, la disfunción motora, la microinflamación intestinal, el estrés o el sobrecrecimiento bacteriano y la microbiota, entre otros. ${ }^{1}$ Si bien existe cierto consenso sobre cuáles son los tratamientos más efectivos, existe un vacío de conocimiento acerca de cuáles son los métodos utilizados por los especialistas en gastroenterología en la Argentina y de cuál es la eficacia considerada para ellos.

El objetivo principal del presente trabajo es describir las modalidades terapéuticas empleadas por los gastroenterólogos en la Argentina y su eficacia en el tratamiento de las variantes del SII. Como objetivo secundario, analizaremos si existen diferencias entre las distintas subespecialidades, la edad y el género de los médicos tratantes.

\section{Materiales y métodos}

Se realizó un estudio de corte transversal a partir de un cuestionario elaborado por expertos. Establecido el objetivo del estudio, se elaboraron los constructos relacionados con los siguientes aspectos vinculados al accionar terapéutico de los gastroenterólogos en las consultas de sus pacientes con SII: datos generales; diferencias entre las diversas variantes del SII (prevalencia, fisiopatología, morbilidad y abordaje terapéutico), y tratamientos farmacológicos y no farmacológicos empleados por los médicos. Para cada estructo o descriptor, se confeccionaron una o varias preguntas. El protocolo fue aprobado por el Comité de Ética del Centro de Educación Médica e Investigaciones Clínicas (CEMIC).

Las encuestas fueron elaboradas en la plataforma Google Forms y enviadas a los médicos gastroenterólogos en la Argentina vía email y mensajería celular. Para su difusión, se contó con el auspicio de sociedades científicas de la especialidad en la Argentina (FAGE, SAGE, CIDE$\mathrm{MO})$. Estimando un número de cuatro mil gastroenterólogos argentinos (unos dos mil socios) y con un margen de error del $5 \%$, se calculó que podría obtenerse un número suficiente de doscientos participantes. El criterio de inclusión de los participantes radicó en ser especialista en gastroenterología y en consentir la participación en el estudio mediante el llenado de la encuesta. Fueron excluidos los médicos no gastroenterólogos.

Las encuestas fueron respondidas en forma anónima y se consideró la respuesta voluntaria como el consentimiento para participar del estudio. Se contabilizó una única respuesta por profesional y se solicitó no repetir el cuestionario si era recibido más de una vez.

Se contó con la participación de colegas representativos de cada una de las áreas densamente pobladas: AMBA: 68\%, Córdoba: 8\%, Rosario: 7\%, Mendoza: 5\%, Tucumán: 4\%, La Plata: 3\%, Mar del Plata: 3\% y Salta: 2\%.

$\mathrm{El}$ análisis estadístico fue efectuado mediante RStudio versión 1.1463. Se empleó el test de t para las variables numéricas continuas de distribución normal y el de chi cuadrado para las variables categóricas. Se describen las variables numéricas como media y desvío estándar y las categóricas como proporciones e intervalo de confianza 95\% (IC 95\%). Se asumió significancia estadística con una $p<0,05$.

\section{Resultados}

Durante el mes de agosto de 2020 se obtuvieron 304 respuestas, 157 de mujeres $(51,6 \%)$ y 147 de hombres $(48,4 \%)$, con una edad media de 44 y 49,9 años (DS \pm 11 $\mathrm{y} \pm 13$ respectivamente, $p<0,001)$. La tasa de respuesta de los participantes fue del 76\% (304 sobre un total de 400 encuestas). Las características de la población se describen en la Tabla 1 y la distribución etaria en la Figura 1. La distribución geográfica de los encuestados se describe en la Figura 2. En la Figura 3 está representada la actividad prioritaria dentro de la especialidad.

Tabla 1. Caracteristicas de los profesionales encuestados

\begin{tabular}{|c|c|c|c|}
\hline & $\begin{array}{l}\text { Hombre } \\
(\mathrm{N}=147)\end{array}$ & $\begin{array}{c}\text { Mujer } \\
(\mathrm{N}=157)\end{array}$ & $p$-valor \\
\hline Edad & & & \\
\hline Media (DS) & $49,9( \pm 12,0)$ & $44,0( \pm 11,0)$ & $<0,001$ \\
\hline $\begin{array}{l}\text { Región de residencia } \\
\text { Centro } \\
\text { Cuyo } \\
\text { NEA } \\
\text { NOA } \\
\text { Patagonia }\end{array}$ & $\begin{array}{c}103(70,07 \%) \\
15(10,20 \%) \\
7(4,76 \%) \\
10(6,80 \%) \\
8(5,44 \%)\end{array}$ & $\begin{array}{c}113(71,97 \%) \\
20(12,74 \%) \\
4(2,55 \%) \\
10(6,37 \%) \\
10(6,37 \%)\end{array}$ & 0,8 \\
\hline $\begin{array}{l}\text { Orientación dicotómica } \\
\text { Neurogastro } \\
\text { No neurogastro }\end{array}$ & $\begin{array}{c}22(14,97 \%) \\
125(85,03 \%)\end{array}$ & $\begin{array}{c}31(19,75 \%) \\
126(80,25 \%)\end{array}$ & 0,3 \\
\hline $\begin{array}{l}\text { Centro médico } \\
\text { Sí } \\
\text { No }\end{array}$ & $\begin{array}{c}35(23,81 \%) \\
112(76,19 \%)\end{array}$ & $\begin{array}{c}51(32,48 \%) \\
106(67,52 \%)\end{array}$ & 0,1 \\
\hline $\begin{array}{l}\text { Consultorio privado } \\
\text { Sí } \\
\text { No }\end{array}$ & $\begin{array}{l}99(67,35 \%) \\
48(32,65 \%)\end{array}$ & $\begin{array}{l}98(62,42 \%) \\
59(37,58 \%)\end{array}$ & 0,4 \\
\hline $\begin{array}{l}\text { Hospital } \\
\text { Sí } \\
\text { No }\end{array}$ & $\begin{array}{l}52(35,37 \%) \\
95(64,63 \%)\end{array}$ & $\begin{array}{l}63(40,13 \%) \\
94(59,87 \%)\end{array}$ & 0,5 \\
\hline
\end{tabular}


Figura 1. Distribución de los profesionales encuestados según género y edad

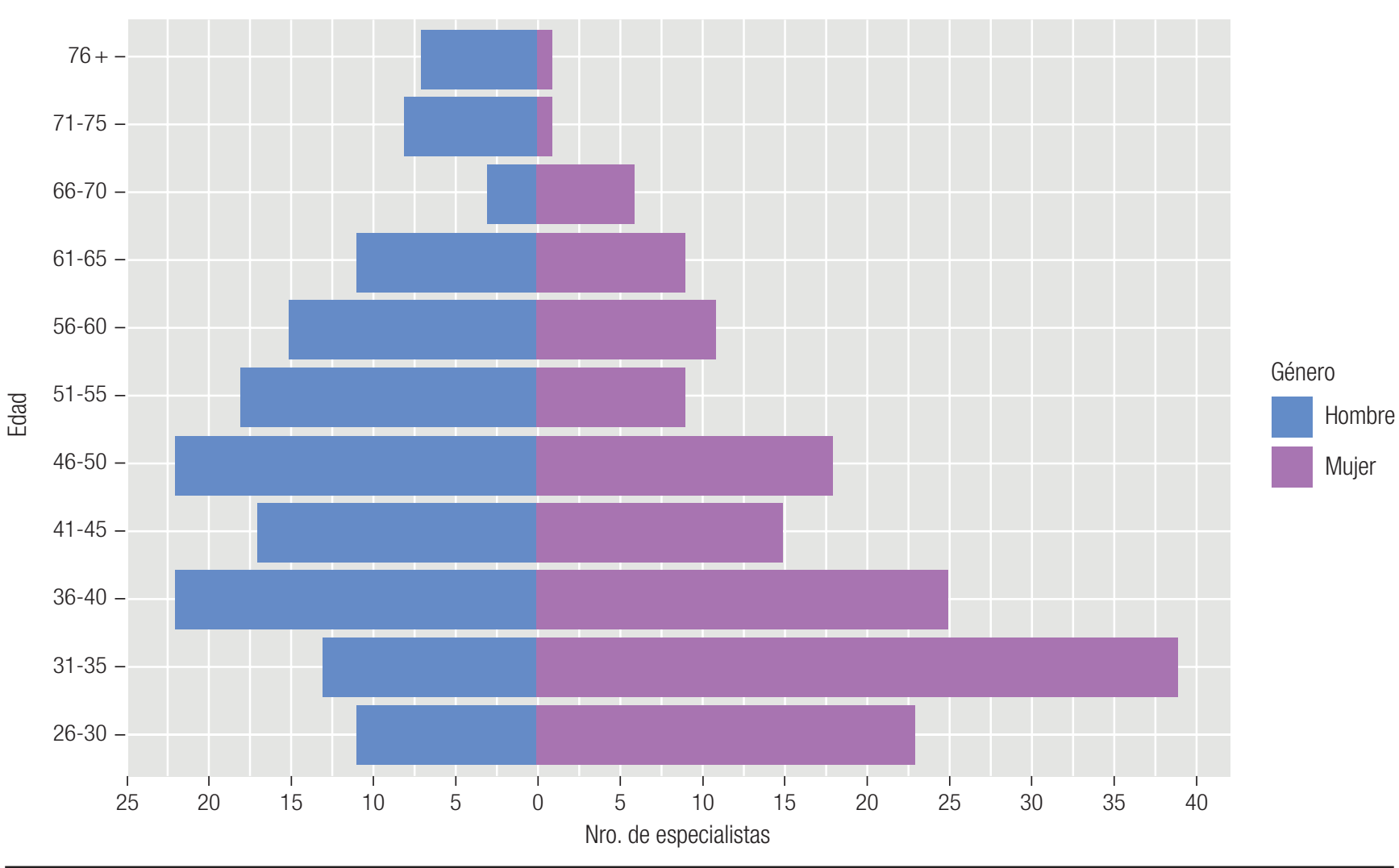

Figura 2. Distribución geográfica de los profesionales encuestados

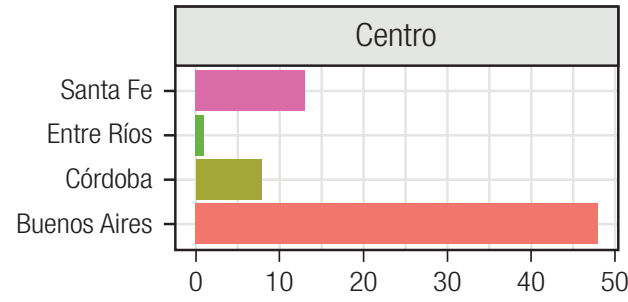

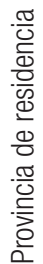
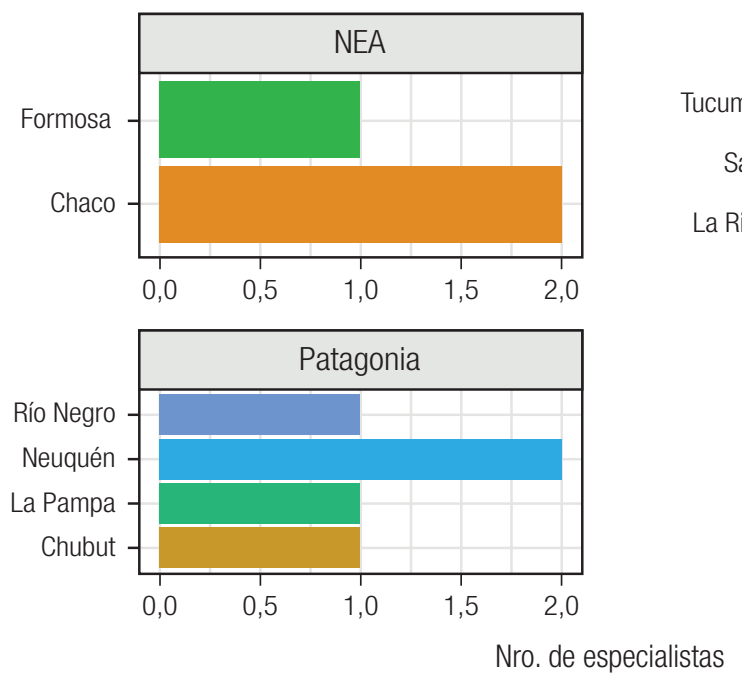

Provincia de residencia
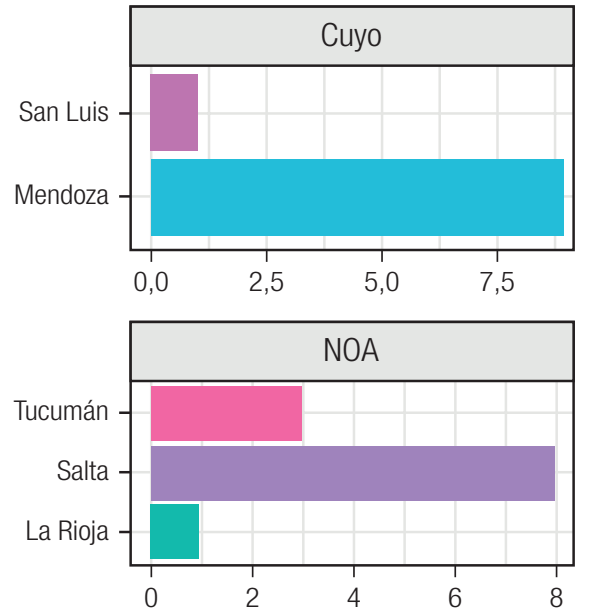

Entre Ríos

Formosa

La Pampa

La Rioja

Mendoza

Neuquén

Río Negro

Salta

San Luis

Santa Fe

Tucumán 
Figura 3. Actividad prioritaria dentro de la especialidad

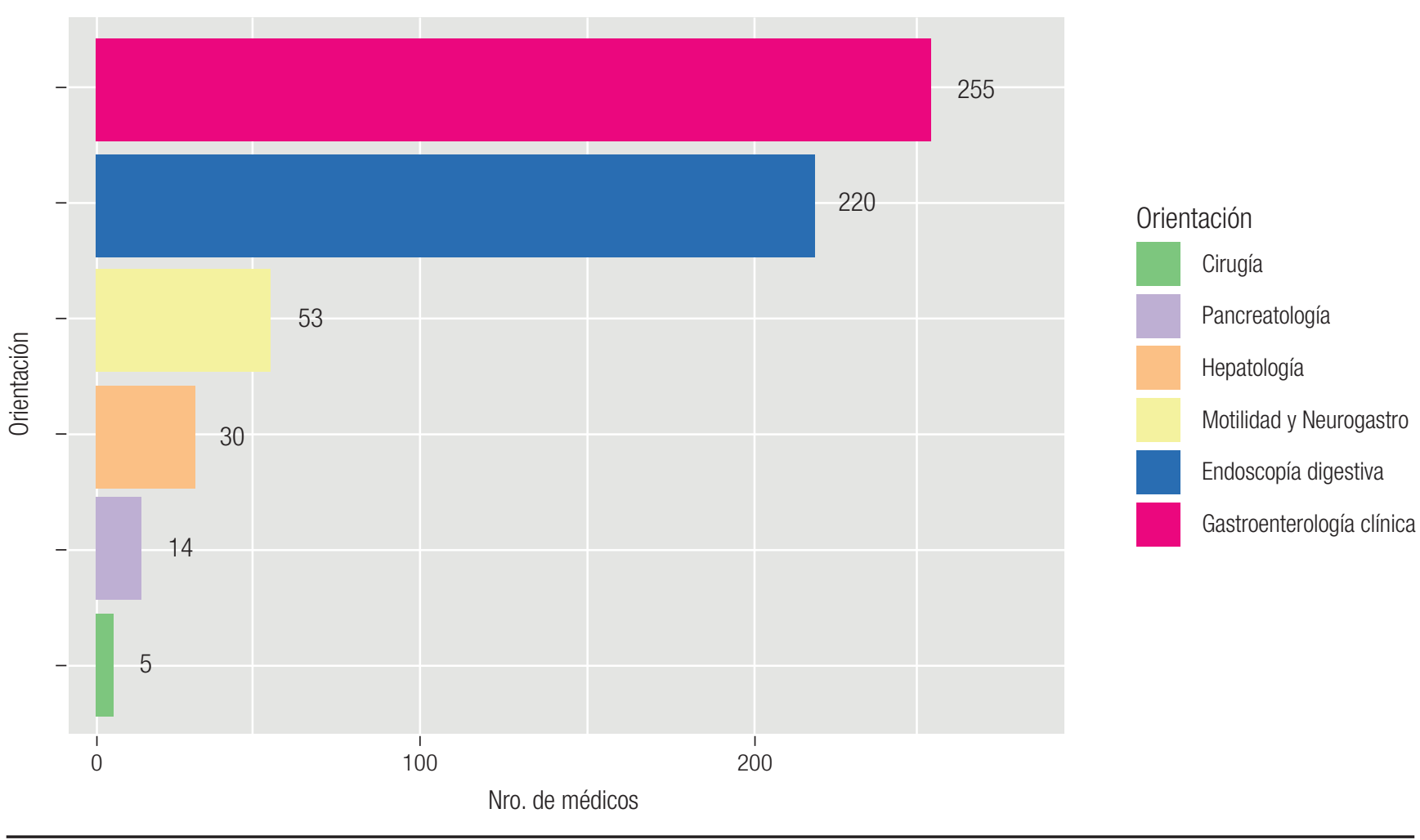

El 48,4\% (147) de los encuestados refiere que los síntomas de SII son el motivo de consulta de los pacientes en el 25 al 50\% de las consultas mensuales. El 33,2\% (101) considera que la proporción es mayor del $50 \%$ y el 18,4\% (56) refiere que es menor del 25\%. Se observaron diferencias estadísticamente significativas en el grupo etario de menos de 40 años, quienes manifestaron una mayor proporción de consultas mensuales $(p<0,01$ para menos de $25 \%$ de consultas mensuales y $p<0,001$ para más del 50\%). No se observaron diferencias significativas con relación a la subespecialidad $(p=0,49$ y $p=0,64$ respectivamente).

En relación con el mecanismo fisiopatológico predominante de cuadro clínico, el 49,3\% (150) se lo atribuye a estrés emocional en el caso de la variante diarrea y el 42,8\% (130) considera la variante constipación como multifactorial, con predominio del tránsito colónico lento (Figura 4).

El $89,6 \%$ (271) de los especialistas consideran que la meta principal del tratamiento del SII es mejorar la calidad de vida de los pacientes, por sobre el alivio sintomático. Si bien no se observaron diferencias estadísticamente significativas con relación a la edad o la subespecialidad ( $p=0,77$ y $p=0,91$ respectivamente), se visualiza una tendencia de los médicos más jóvenes hacia la búsqueda de una mejoría en la calidad de vida como meta principal del tratamiento (Figura 5). La variante alternante fue considerada la que presenta menos recursos terapéuticos, siendo referida por el 44,4\% (135) de los encuestados vs. $28,6 \%$ (87) para el SII-D y $14,1 \%$ (43) para el SII-C. Un 12,8\% (39) no manifiesta diferencias entre variantes en relación con los recursos disponibles. En cuanto a la efectividad de los diversos tratamientos, en la Tabla 2 se describen los empleados en el SII-D y en la Tabla 3 los utilizados en el SII-C. El 96,1\% (292) de los gastroenterólogos consideran la rifaximina como un tratamiento efectivo para el SII-D, seguida por los antiespasmódicos, la loperamida y la colestiramina (Tabla 2). Con respecto al SII-C, el 96,7\% (294) observa efectividad con el polietilenglicol, seguido de la linaclotida, el psyllium y el prucalopride (Tabla 3). Se observaron entre los especialistas diferencias estadísticamente significativas en el tratamiento del SII-D respecto de la efectividad considerada para la dieta $(p=0,05)$, la amitriptilina $(p=0,007)$ y el uso de antibióticos distintos de la rifaximina $(p<0,001)$. En el manejo del SII-C, se encontraron diferencias estadísticamente significativas en la efectividad considerada para el uso de bisacodilo/picosulfato y para los antibióticos $(p=0,004)$. 
Figura 4. Fisiopatología del SII según la orientación del especialista: (A) variante diarrea, (B) variante constipación.

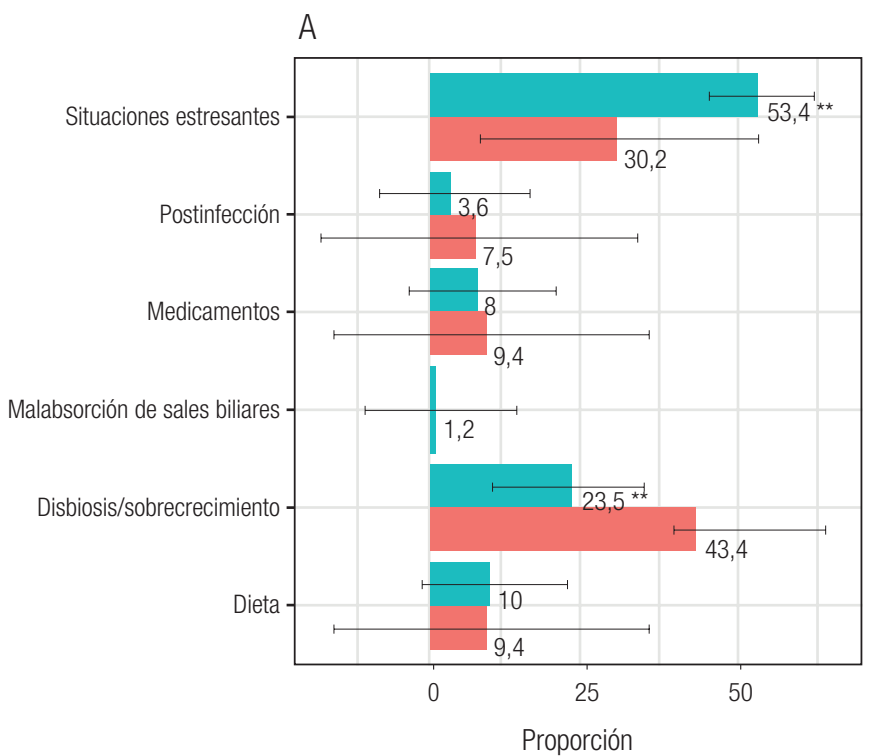

Neurogastro

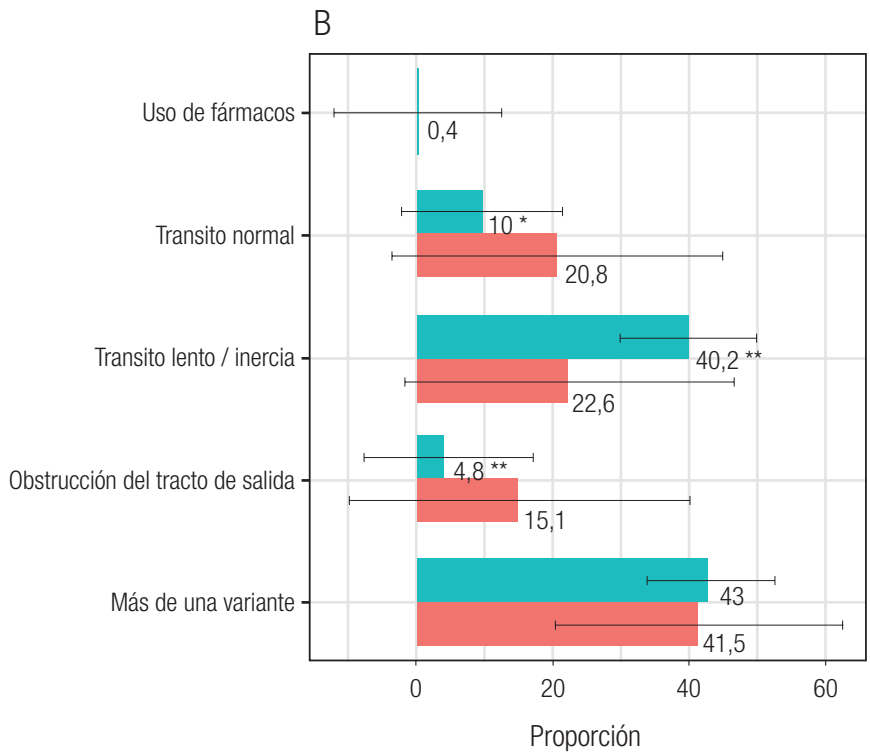

No neurogastro

${ }^{\star} p=0,05 ;{ }^{* *} p<0,05$.

Figura 5. Metas en el tratamiento del SII en función de la edad

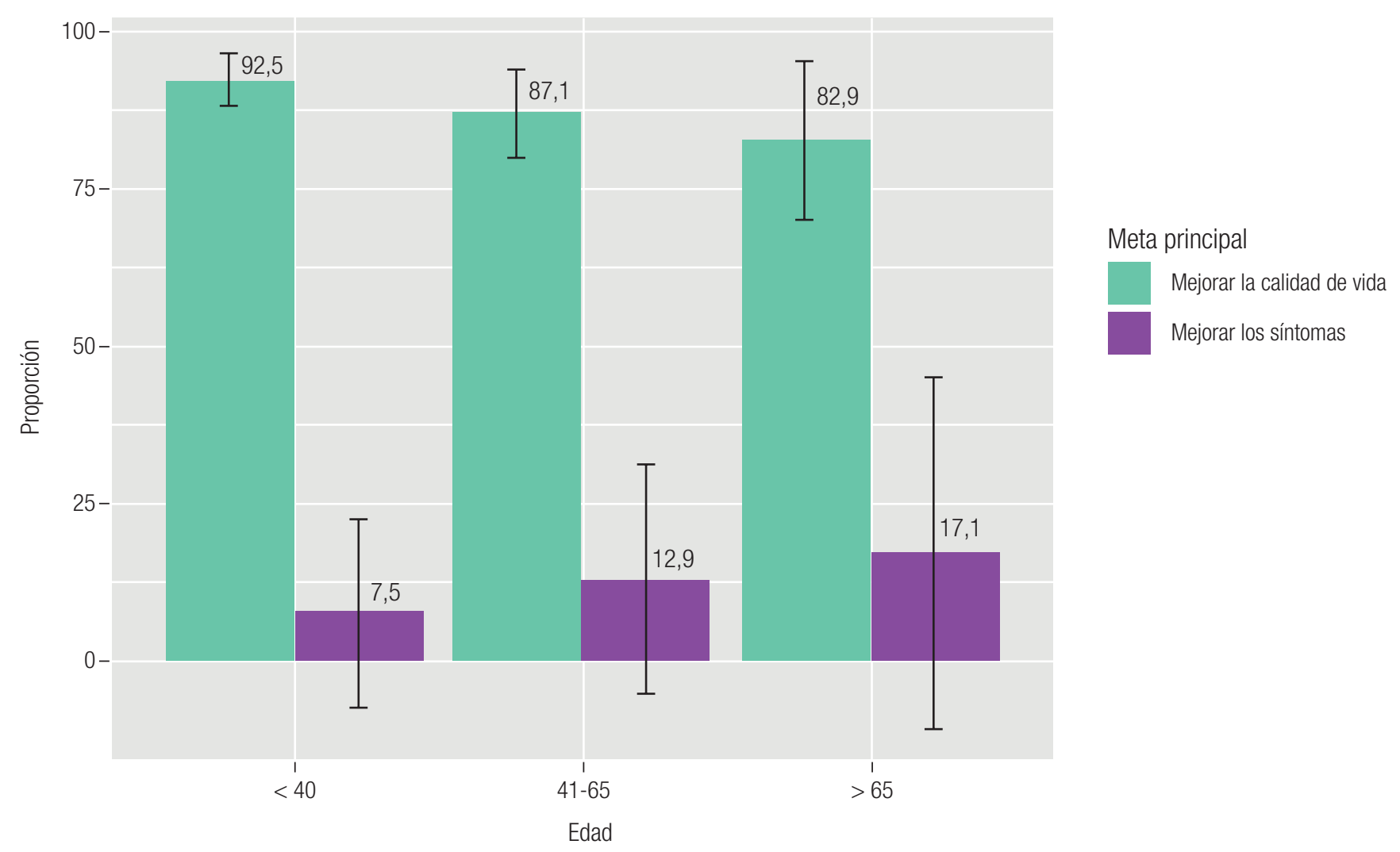


Tabla 2. Efectividad considerada para los diversos tratamientos en el SII-D

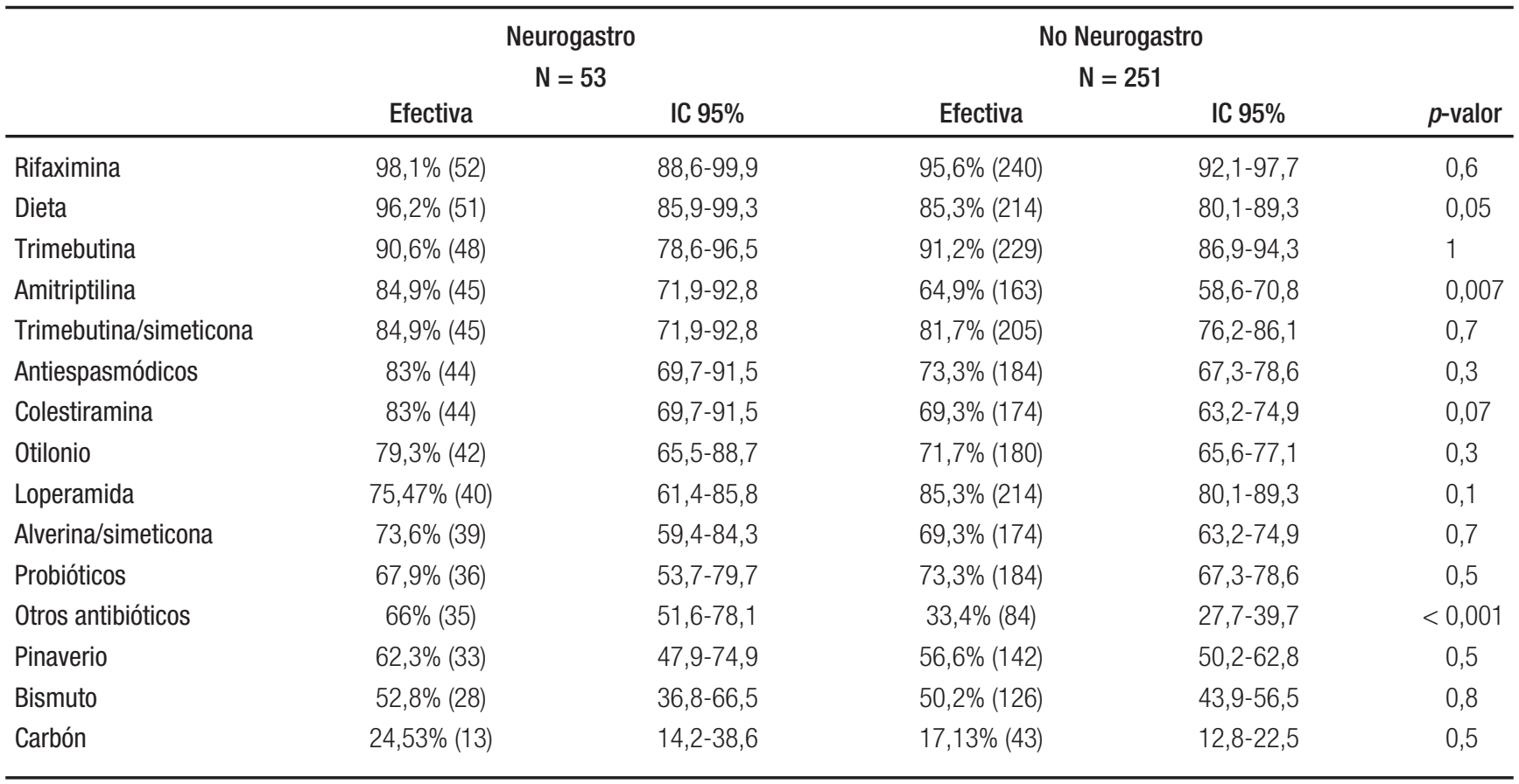

Tabla 3. Efectividad considerada para los diversos tratamientos en el SII-C

\begin{tabular}{|c|c|c|c|c|c|}
\hline & \multicolumn{2}{|c|}{$\begin{array}{c}\text { Neurogastro } \\
\mathrm{N}=53\end{array}$} & \multicolumn{2}{|c|}{$\begin{array}{c}\text { No Neurogastro } \\
N=251\end{array}$} & \multirow[b]{2}{*}{$p$-valor } \\
\hline & Efectiva & IC 95\% & Efectiva & IC 95\% & \\
\hline Polietilenglicol & $100,0 \%(53)$ & $91,6-100$ & $96 \%(241)$ & $92,6-98$ & 0,3 \\
\hline Linaclotida & $92,5 \%(49)$ & $80,9-97,6$ & $88,8 \%(223)$ & $84,1-91,3$ & 0,6 \\
\hline Dieta & $90,6 \%(48)$ & $78,6-96,5$ & $92 \%(231)$ & $87,8-94,9$ & 0,9 \\
\hline Psyllium & $88,7 \%(47)$ & $76,3-95,3$ & $87,3 \%(219)$ & $82,3-91$ & 1 \\
\hline Prucalopride & $84,9 \%(45)$ & $71,9-92,8$ & $81,3 \%(204)$ & $75,8-85,8$ & 0,7 \\
\hline Bisacodilo/picosulfato & $79,3 \%(42)$ & $65,5-88,7$ & $56,6 \%(142)$ & $50,2-62,8$ & 0,004 \\
\hline Lactulosa & $62,3 \%(33)$ & $47,8-74,9$ & $69,3 \%(174)$ & $63,2-74,9$ & 0,4 \\
\hline Antibióticos & $58,5 \%(31)$ & $44,2-71,6$ & $35,9 \%(90)$ & $30-42,1$ & 0,004 \\
\hline Trimebutina & $50,9 \%(27)$ & $37-64,7$ & $40,2 \%(101)$ & $34,2-46,6$ & 0,2 \\
\hline Sales biliares & $45,3 \%(24)$ & $31,8-59,4$ & $39,4 \%(99)$ & $33,4-45,8$ & 0,5 \\
\hline Senósidos & $45,3 \%(24)$ & $31,8-59,4$ & $40,6 \%(102)$ & $34,6-47$ & 0,6 \\
\hline
\end{tabular}

El 65,6\% (198) de los encuestados prefieren en una primera instancia evitar la medicación y emplear dietas y productos naturales. El 53\% (160) se sienten seguros de prescribir antidepresivos, mientras que el 41,7\% (126) considera que este tipo de fármacos deben ser indicados por un psiquiatra. Un 5,3\% (16) nunca consideró el empleo de este tipo de medicación en el SII. En relación con las terapias no farmacológicas, un 93\% (283) considera útil recurrir a psicoterapia, un 46,7\% (142) al yoga, un $37,8 \%(115)$ a la meditación, un $30,6 \%(93)$ a mindful- ness y un 6,9\% (21) a hipnoterapia. Se observaron diferencias estadísticamente significativas entre neurogastroenterólogos y el resto de los especialistas en relación con el uso de hipnoterapia $(23,53 \%$ vs. $3,19 \%$ respectivamente; $p<0,001)$.

\section{Discusión}

El SII constituye uno de los motivos de consulta más frecuentes y relevantes en la práctica gastroenterológica y representa una importante demanda de recursos tanto 
humanos como materiales. Si bien es un trastorno benigno, el compromiso en la calidad de vida resulta significativo y la evidencia científica demuestra que la buena relación médico-paciente se asocia con un descenso en el número de consultas, el alivio sintomático y el incremento de la satisfacción del paciente. ${ }^{4}$ En nuestro trabajo observamos que la gran mayoría de los gastroenterólogos en la Argentina tiene como meta principal del tratamiento la mejoría en la calidad de vida, por sobre la atenuación de los síntomas, y que existe una tendencia mayor hacia este objetivo cuanto más joven es el médico tratante. En esta búsqueda, una gran proporción de los especialistas prefiere iniciar el abordaje terapéutico empleando dietas y medidas generales con el propósito de lograr cambios en el estilo de vida y fomentar hábitos más saludables. La evidencia científica demuestra que muchas de estas acciones tienen una eficacia probada..$^{5-9}$ De acuerdo con las guías para el manejo del SII elaboradas por el Colegio Americano de Gastroenterología (ACG, por sus siglas en inglés), se recomienda realizar ejercicio físico y una dieta baja en FODMAPs para la mejoría global de los síntomas (recomendación débil, evidencia muy baja). ${ }^{9}$

Según nuestra encuesta, casi un 50\% de los gastroenterólogos en la Argentina considera el estrés como el mecanismo fisiopatológico más prevalente en el SII-D. Acorde con ello, el 93\% suele recomendar a sus pacientes algún tipo de apoyo psicológico. Sin embargo, en el empleo de tratamiento farmacológico, una mayor proporción de especialistas señala la rifaximina como el medicamento de mayor efectividad y casi la mitad de los especialistas manifiesta que los antidepresivos deberían ser indicados por médicos psiquiatras. En una revisión sistemática sobre la eficacia del tratamiento con psicoterapia (cognitivo-conductual y cuerpo-mente) en pacientes con SII, se observó que esta disminuye la intensidad de los síntomas globales, y en particular la del dolor abdominal, y que mejora la calidad de vida de los pacientes. ${ }^{10}$ Según las guías del ACG, se sugiere algún tipo de terapia psicológica para la mejoría global de los síntomas en los pacientes con SII (recomendación débil, evidencia muy baja). ${ }^{9}$ En cuanto al empleo de antidepresivos, los inhibidores selectivos de la recaptación de serotonina muestran eficacia para la mejoría global de los síntomas, mientras que los antidepresivos tricíclicos son eficaces para la mejoría del dolor. ${ }^{11} \mathrm{El}$ ACG recomienda el uso de antidepresivos tricíclicos para la mejoría global de los síntomas (recomendación fuerte, evidencia alta) y sugiere el uso de inhibidores selectivos de la recaptación de serotonina para la mejoría global de los síntomas (recomendación débil, evidencia baja).?

En relación con la eficacia del tratamiento farmacológico en el SII-D, la elección de la rifaximina como la me- dicación de mayor efectividad $(96,1 \%)$ implicaría considerar la microbiota, y especialmente el sobrecrecimiento bacteriano, como un factor relevante en la fisiopatología de los síntomas. Dos metaanálisis han evaluado la eficacia de la rifaximina en el SII. Li et al. concluyeron que es superior al placebo en la mejoría global de los síntomas, especialmente en el alivio de la distensión abdominal. ${ }^{12}$ En concordancia con este trabajo, Menees et al. obtuvieron hallazgos similares en cuanto a la mejoría global de los síntomas y de la distensión abdominal. ${ }^{13}$ Asimismo, en un estudio reciente, Lembo et al. demostraron la eficacia del tratamiento repetido con rifaximina. ${ }^{14}$ En cuanto a la tolerancia y los efectos adversos de este fármaco, se ha concluido que no hay diferencias con respecto al placebo. ${ }^{15}$ En conclusión, y en concordancia con nuestros hallazgos, la rifaximina es un fármaco seguro y eficaz en pacientes con SII sin estreñimiento y resulta especialmente útil en pacientes en los que predomina la distensión. Según las guías del ACG, se recomienda el uso de rifaximina para la mejoría global de los síntomas, así como también para la distensión en pacientes con SII sin constipación (recomendación débil, evidencia moderada). ${ }^{9}$

La eficacia considerada para los antiespasmódicos varía de acuerdo al fármaco utilizado. La trimebutina y sus combinaciones con antiflatulentos o ansiolíticos resulta la más eficaz, siendo referida como efectiva para el tratamiento del SII-D por más del $90 \%$ de los gastroenterólogos en la Argentina. El mecanismo de acción de estos fármacos es la inhibición de la motilidad intestinal por antagonismo de receptores $\mu$, de receptores muscarínicos M3, o por el bloqueo de los canales del calcio. Su eficacia en el tratamiento del SII se ha evaluado en tres metaanálisis, observándose mejoría global de los síntomas, en especial del dolor abdominal. ${ }^{11,16,17}$ Según la Secretaría de Programación para la Prevención de la Drogadicción y Lucha contra el Narcotráfico (SEDRONAR), el empleo de ansiolíticos para diversos cuadros clínicos está ampliamente difundido en el país. ${ }^{18}$ No obstante, la prescripción de estos fármacos debe realizarse con precaución, ya que se han observado efectos adictivos y alteraciones cognitivas cuando son empleados en forma crónica. ${ }^{19}$ De acuerdo con nuestro trabajo, los gastroenterólogos en la Argentina, encuentran una mayor eficacia en la combinación de ansiolíticos con antiespasmódicos que en el empleo aislado de antiespasmódicos. En dosis bajas y por períodos cortos, los ansiolíticos serían útiles en aquellos pacientes en los que cuales el estrés tiene una clara vinculación con la reagudización sintomática. ${ }^{20}$ El ACG sugiere el uso de ciertos antiespasmódicos (otilonio, pinaverio o hioscina, entre otros) para la mejoría global de los sín- 
tomas en los pacientes con SII (recomendación débil, evidencia muy baja).?

La loperamida es referida como efectiva para el tratamiento del SII-D por el $83,5 \%$ de los encuestados, después de la dieta y de la trimebutina. Se trata de un agonista de los receptores $\mu$ opioides, cuyo mecanismo de acción consiste en enlentecer el tránsito intestinal. Su eficacia ha sido evaluada frente a placebo en dos ensayos clínicos con un tamaño muestral pequeño, y se evidenció eficacia en la mejoría de la diarrea, sin alivio de los síntomas globales ni del dolor. ${ }^{21,22}$ La dosis necesaria para un adecuado control de la diarrea resulta variable entre pacientes y debe usarse con precaución en aquellos con síntomas alternantes. ${ }^{23}$ Aunque la evidencia es de baja calidad, se recomienda su uso en pacientes con SII-D, especialmente si se asocia con urgencia defecatoria e incontinencia. Sin embargo, debería tenerse en cuenta que el enlentecimiento del tránsito podría contribuir a promover el sobrecrecimiento bacteriano. De acuerdo con las guías del ACG, se recomienda en contra del uso de loperamida para la mejoría de los síntomas del SII (recomendación fuerte, evidencia muy baja). ${ }^{9}$

Los probióticos, por otro lado, son reconocidos como útiles en el tratamiento del SII-D por el 72,3\% de los especialistas. De acuerdo con una revisión sistemática y un metaanálisis, su uso se asocia a una mejoría significativa de la percepción global de los síntomas en pacientes con SII, principalmente respecto del dolor, la distensión y el meteorismo. Sin embargo, no hay datos concluyentes acerca de su influencia en el estreñimiento. ${ }^{24}$ El ACG aconseja su uso para la mejoría global de los síntomas, así como también para la distensión y la flatulencia en los pacientes con SII (recomendación débil, evidencia baja). ${ }^{9}$

Tampoco existen datos concluyentes en relación con el uso de colestiramina en el SII. El fundamento de su utilización radica en que su mecanismo de acción es la adsorción de sales biliares, lo cual disminuiría su efecto estimulante sobre la motilidad colónica. Si bien se ha observado una alta prevalencia de malabsorción de ácidos biliares en los pacientes con SII-D, no existen aún estudios controlados que avalen su empleo. ${ }^{25}$ En nuestro trabajo, observamos que el 71,7\% de los encuestados la considera efectiva en el tratamiento del SII-D. No obstante, no hemos hallado en la bibliografía ninguna recomendación forma sobre su uso en el SII.

Con respecto al tratamiento del SII-C, el polietilenglicol 3350 (PEG) fue la medicación que se consideró más efectiva (96,7\%). El PEG es el único laxante en el tratamiento del SII-C que se ha evaluado contra placebo en ensayos clínicos aleatorizados, aunque el seguimiento en estos estudios fue relativamente corto (cuatro semanas).
Awad et al. Observaron una mejoría del estreñimiento y de los síntomas asociados (dolor, meteorismo y distensión), sin hallar diferencias significativas respecto del placebo. ${ }^{26}$ Contrario a esto, Chapman et al. evidenciaron que el PEG fue superior al placebo en mejorar el estreñimiento, pero no así el dolor y la distensión abdominal. ${ }^{27}$ Comparativamente, el PEG ha evidenciado ser más eficaz que la lactulosa ${ }^{28}$ y puede administrarse durante largos períodos de tiempo, con un buen perfil de seguridad. ${ }^{29}$ De acuerdo con las guías del ACG, se desaconseja el uso de PEG para la mejoría global de los síntomas en los pacientes con SII (recomendación débil, evidencia muy baja).

La linaclotida es considerada efectiva por el $89,5 \%$ de los encuestados. Este fármaco es un agonista de la guanilato ciclasa $C$, que actúa aumentando la secreción de bicarbonato y cloro hacia la luz intestinal. Incrementa el contenido intestinal, acelera el tiempo de tránsito y disminuye la sensibilidad visceral. ${ }^{30,31}$ Los ensayos clínicos y metaanálisis en pacientes con SII-C que comparan linaclotida contra placebo demuestran una significativa superioridad de esta droga respecto tanto del estreñimiento como de los síntomas asociados (dolor y distensión abdominal). ${ }^{32,33}$ La linaclotida presenta una escasa a nula absorción intestinal, resulta segura a cualquier edad y sus efectos sobre el tránsito intestinal suelen ser evidentes en forma temprana, durante la primera semana de tratamiento. Sin embargo, su beneficio sobre el dolor y la distensión puede ser tardío, por lo que se recomienda un mínimo de 4-10 semanas de tratamiento. ${ }^{34}$ El ACG recomienda el uso de la linaclotida para la mejoría global de los síntomas en los pacientes con SII-C (recomendación fuerte, evidencia alta). ${ }^{\text {? }}$

La fibra soluble (Psyllium), considerada efectiva por el $87,5 \%$, ha demostrado ser beneficiosa en la mejoría del estreñimiento en el SII-C, pero puede exacerbar el dolor abdominal, la distensión y el meteorismo. Se recomienda su empleo en forma progresiva, hasta alcanzar una dosis diaria de 20 a 30 gramos. ${ }^{35-37}$ Por otro lado, los laxantes estimulantes (bisacodilo y picosulfato) fueron considerados efectivos por el $60,5 \%$. Si bien existe evidencia de su utilidad en el estreñimiento, no han sido evaluados específicamente en pacientes con SII-C. Además, estos fármacos presentan como efectos adversos secundarios el dolor abdominal, la distensión y la diarrea, por lo que deberían emplearse con precaución en pacientes con SII. ${ }^{38,39}$ Según las guías del ACG, se recomienda una dieta rica en fibra y el uso de psyllium para la mejoría global de los síntomas (recomendación fuerte, evidencia moderada). ${ }^{9}$

En el caso del prucalopride, su estimulación selectiva de los receptores $5 \mathrm{HT} 4$ estimula la motilidad intestinal y 
la liberación de agua por parte de los colonocitos, siendo la acción más efectiva cuanto mayor sea la densidad de receptores a nivel colónico. ${ }^{40}$ Aunque aún no se han realizado estudios que evalúen la eficacia de este fármaco en el SII-C, se espera que resulte eficaz para los síntomas de la enfermedad. Sin embargo, el agravamiento del dolor abdominal podría limitar su uso en la práctica clínica. ${ }^{40} \mathrm{De}$ acuerdo con nuestra encuesta, el 81,9\% de los especialistas en la Argentina refiere su efectividad en el tratamiento del SII-C. No hemos hallado en la bibliografía ninguna recomendación formal sobre el uso de prucalopride en el SII, como así tampoco evidencia científica que avale su indicación en el SII.

Con respecto a las terapias alternativas, un $46,7 \%$ de los encuestados manifestaron la utilidad del yoga. En una revisión sistemática de seis estudios controlados, se observaron efectos beneficiosos en relación con la reducción de la severidad sintomática y de la ansiedad, así como cierta mejoría en la calidad de vida. Sin embargo, debido a la diversidad de las metodologías empleadas, la evidencia resulta insuficiente para poder efectuar una recomendación. ${ }^{41}$ Asimismo, un 37,8\% y un 30,6\% de los encuestados refirieron útiles la meditación y el mindfulness respectivamente. En relación con estos hallazgos, en un metaanálisis de seis estudios controlados, se observaron mejorías significativas en relación con el alivio sintomático y la calidad de vida. Sin embargo, los autores refieren que dichos estudios presentaban un riesgo alto de sesgo. Si bien existe cierta evidencia que sugiere que las intervenciones basadas en la atención plena pueden proporcionar beneficios en los trastornos gastrointestinales funcionales, se necesitan mejoras sustanciales en la calidad metodológica de estos trabajos para poder efectuar recomendaciones. ${ }^{42} \mathrm{Si}$ bien la hipnosis no resulta un método considerado efectivo entre los gastroenterólogos en la Argentina, algunos trabajos sugieren una eficacia similar a la de los antidepresivos y la psicoterapia. ${ }^{43}$

Existen pocos trabajos similares al nuestro en la bibliografía. De acuerdo con una encuesta realizada entre gastroenterólgos italianos por Soncini et al., ${ }^{44}$ aproximadamente el $70 \%$ de ellos sugiere a los pacientes cambios en el estilo de vida y en la dieta, mientras que un 50\% indica probióticos. En relación con los subtipos de SII, el polietilenglicol, la suplementación con fibras y la linaclotida son los más frecuentemente indicados en el SII-C. Finalmente, los autores concuerdan que el abordaje terapéutico del SII resulta variado y francamente heterogéneo, y que el manejo de esta condición resulta todavía ampliamente inapropiado. Nuestras observaciones resultan similares a las mencionadas por los autores, lo cual nos sugiere que las discrepancias y dificultades en el tratamiento del SII no resultan exclusivas de los gastroenterólogos argentinos.

La principal fortaleza de nuestro trabajo radicó en la posibilidad de elaborar una encuesta que logró ser distribuida en todo el territorio argentino, permitiendo una representatividad aceptable de los gastroenterólogos del país. Asimismo, las respuestas pudieron ser analizadas de acuerdo con el grupo etario y la subespecialidad. Con respecto a esto último, se observaron diferencias estadísticamente significativas en relación con el tratamiento cuando se compararon las respuestas de los neurogastroenterólogos en relación con el resto de los especialistas.

Con respecto a las limitaciones, si bien encontramos ciertas tendencias en algunas respuestas, muchas de ellas no resultaron estadísticamente significativas, lo que podría estar relacionado con el tamaño de la muestra. Por otro lado, debido a la imposibilidad de contacto directo con los colegas, a causa de las restricciones asociadas a la pandemia, nos vimos limitados a distribuir la encuesta en forma virtual, a través de los canales mencionados, lo cual podría resultar en un sesgo de reclutamiento de los encuestados. Finalmente, la interpretación del empleo y de la eficacia de diversos tratamientos empleados en los pacientes con SII puede estar sesgada por la influencia directa o indirecta que ejerce la industria farmacéutica en las opiniones de los médicos encuestados, así como también por la diferencia de apreciación subjetiva entre los distintos profesionales con relación al grado de eficacia de los diversos tratamientos.

\section{Conclusión}

La gran mayoría de los encuestados considera que la meta principal del tratamiento es la mejoría de la calidad de vida, por sobre el alivio sintomático. Es mayoritaria la postura de iniciar el tratamiento con dieta y medidas generales orientadas al refuerzo de hábitos saludables, antes de prescribir esquemas farmacológicos. Si bien ciertas drogas son consideradas efectivas en el tratamiento de las distintas variantes del SII, ninguna de ellas es referida como muy efectiva por al menos el $50 \%$ de los encuestados. Tampoco lo fueron las terapias no farmacológicas.

El mecanismo fisiopatológico considerado más frecuente en el SII-D es el estrés. Sin embargo, la medicación considerada más efectiva para esta variante es la rifaximina, la cual actúa modulando la microbiota intestinal.

Se observaron diferencias estadísticamente significativas en la proporción de consultas mensuales por SII con relación a la edad y en la efectividad considerada 
para algunos tratamientos en relación con la subespecialidad de los médicos encuestados.

El abordaje terapéutico del SII por los gastroenterólogos argentinos es variado y algo heterogéneo, lo cual resulta en un manejo todavía inapropiado de esta condición.

\section{Conflictos de interés. Ninguno de los autores manifiesta la existencia de conflictos de interés.}

Consentimiento para la publicación. Para la confección de este manuscrito, se utilizaron datos anonimizados que no han distorsionado su significado científico.

Propiedad intelectual. Los autores declaran que los datos, las figuras y las tablas presentes en el manuscrito son originales y fueron realizados en sus instituciones pertenecientes.

\section{Aviso de derechos de autor}

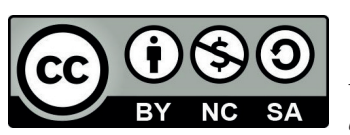

(C) 2021 Acta Gastroenterológica Latinoamericana. Este es un artículo de acceso abierto publicado bajo los términos de la Licencia Creative Commons Attribution (CC BY-NC-SA 4.0), la cual permite el uso, la distribución $y$ la reproducción de forma no comercial, siempre que se cite al autor y la fuente original.

Cite este artículo como: Soifer L, Tawil J, Rey A y col. Tratamiento del sindrome de intestino irritable por gastroenterólogos: resultados de un estudio de corte transversal a partir de una encuesta elaborada por expertos. Acta Gastroenterol Latinoam. 2021;51(3):280-91. https://doi.org/10.52787/ $k k s \times 5531$

\section{Referencias}

1. Balboa A, Ciriza C, Delgado-Aros S, Fernandez-Bañarez F, Mearin F, Mínguez M, y cols. Guía AEG - síndrome del intestino irritable. 2017:1-88.

2. Usai-Satta P, Bellini M, Lai M, Oppia F, Cabras F. Therapeutic Approach for Irritable Bowel Syndrome: Old and New Strategies. Curr Clin Pharmacol. 2018;13(3):164-72.

3. Palsson OS, Whitehead WE, Van Tilburg MAL, Chang L, Chey W, Crowell MD, et al. Development and validation of the Rome IV diagnostic questionnaire for adults. Gastroenterology. 2016;150(6):1481-91.

4. Owens DM, Nelson DK, Talley NJ. The irritable bowel syndrome: Long-term prognosis and the physician- patient interaction. Ann Intern Med. 1995;122(2):107-12.

5. Ong DK, Mitchell SB, Barrett JS, Shepherd SJ, Irving PM, Biesiekierski JR, et al. Manipulation of dietary short chain carbohydrates alters the pattern of gas production and genesis of symptoms in irritable bowel syndrome. J Gastroenterol Hepatol. 2010;25(8):1366-73.
6. De Roest RH, Dobbs BR, Chapman BA, Batman B, O'Brien LA, Leeper JA, et al. The low FODMAP diet improves gastrointestinal symptoms in patients with irritable bowel syndrome: A prospective study. Int J Clin Pract. 2013;67(9):895-903.

7. Biesiekierski JR, Peters SL, Newnham ED, Rosella O, Muir JG, Gibson PR. No Effects of Gluten in Patients With Self-Reported Non-Celiac Gluten Sensitivity After Dietary Reduction of Fermentable, Poorly Absorbed, Short-Chain Carbohydrates. Gastroenterology. 2013;145(2):320-8.e3.

8. Halmos EP, Power VA, Shepherd SJ, Gibson PR, Muir JG. A diet low in FODMAPs reduces symptoms of irritable bowel syndrome. Gastroenterology. 2014;146(1).

9. Ford AC, Moayyedi P, Chey WD, Harris LA, Lacy BE, Saito YA, et al. American college of gastroenterology monograph on management of irritable bowel syndrome. Am J Gastroenterol. 2018;113(S2):1-18.

10. Altayar O, Sharma V, Prokop LJ, Sood A, Murad MH. Psychological Therapies in Patients with Irritable Bowel Syndrome: A Systematic Review and Meta-Analysis of Randomized Controlled Trials. Gastroenterology Research and Practice. Hindawi Limited. 2015;2015.

11. Ruepert L, Quartero AO, de Wit NJ, Van der Heijden GJ, Rubin G, Muris JW. Bulking agents, antispasmodics and antidepressants for the treatment of irritable bowel syndrome. Cochrane Database of Systematic Reviews. John Wiley and Sons Ltd. 2011;2011.

12. Li J, Zhu W, Liu W, Wu Y, Wu B. Rifaximin for irritable bowel syndrome: A Meta-Analysis of randomized placebo-controlled trials. Vol. 95, Medicine (EE. UU). Lippincott Williams and Wilkins. 2016.

13. Menees SB, Maneerattannaporn M, Kim HM, Chey WD. The efficacy and safety of rifaximin for the irritable bowel syndrome: A systematic review and meta-analysis. American Journal of Gastroenterology. Am J Gastroenterol. 2012;107:28-35.

14. Lembo A, Pimentel M, Rao SS, Schoenfeld P, Cash B, Weinstock LB, et al. Repeat Treatment With Rifaximin Is Safe and Effective in Patients With Diarrhea-Predominant Irritable Bowel Syndrome. Gastroenterology. 2016;151(6):1113-21.

15. Schoenfeld P, Pimentel M, Chang L, Lembo A, Chey WD, Yu $\mathrm{J}$, et al. Safety and tolerability of rifaximin for the treatment of irritable bowel syndrome without constipation: A pooled analysis of randomised, double-blind, placebo-controlled trials. Aliment Pharmacol Ther. 2014;39(10):1161-8.

16. Lesbros-Pantoflickova D, Michetti P, Fried M, Beglinger C, Blum AL. Meta-analysis: The treatment of irritable bowel syndrome. Alimentary Pharmacology and Therapeutics. Aliment Pharmacol Ther. 2004;20:1253-69.

17. Ford AC, Talley NJ, Spiegel BMR, Foxx-Orenstein AE, Schiller L, Quigley EMM, et al. Effect of fibre, antispasmodics, and peppermint oil in the treatment of irritable bowel syndrome: Systematic review and meta-analysis. BMJ. 2008;337:1388-92.

18. Secretaría de Programación para la Prevención de la Drogadicción y Lucha contra el Narcotráfico. Consumo de Psicofármacos: Estudio Nacional en Población de 12 a 65 años sobre Consumo de Sustancias Psicoactivas. Argentina. 2017;41.

19. Sprung DJ. Risk of Addiction For IBS Patients Prescribed Anxiolytics: A Official journal of the American College of Gastroenterology | ACG. Am J Gastroenterol. 2017;112:228.

20. Dell'osso B, Lader M. Do benzodiazepines still deserve a major role in the treatment of psychiatric disorders? A critical reappraisal. European Psychiatry. Eur Psychiatry. 2013;28:7-20. 
21. Lävo B, Stenstam M, Nielsen AL. Loperamide in treatment of irritable bowel syndrome - a double-blind placebo controlled study. Scand J Gastroenterol. 1987;22(S130):77-80.

22. Efskind PS, Bernklev T, Vatn MH. A double-blind placebo-controlled trial with loperamide in irritable bowel syndrome. Scand J Gastroenterol. 1996;31(5):463-8.

23. Ragnarsson G, Bodemar G. Treatment of irritable bowel syndrome with loperamide oxide. An open study to determine optimal dosage. Journal of Internal Medicine. J Intern Med. 2000;248:165-6.

24. Ford AC, Quigley EMM, Lacy BE, Lembo AJ, Saito YA, Schiller LR, et al. Efficacy of prebiotics, probiotics, and synbiotics in irritable bowel syndrome and chronic idiopathic constipation: systematic review and meta-analysis. Am J Gastroenterol. 2014;109(10):1547-62.

25. Wedlake L, A'Hern R, Russell D, Thomas K, Walters JRF, Andreyev HJN. Systematic review: The prevalence of idiopathic bile acid malabsorption as diagnosed by SeHCAT scanning in patients with diarrhoea-predominant irritable bowel syndrome. Aliment Pharmacol Ther. 2009;30(7):707-17.

26. Awad RA, Camacho S. A randomized, double-blind, placebo-controlled trial of polyethylene glycol effects on fasting and postprandial rectal sensitivity and symptoms in hypersensitive constipation-predominant irritable bowel syndrome. Colorectal Dis. 2010;12(11):1131-8.

27. Chapman RW, Stanghellini V, Geraint M, Halphen M. Randomized clinical trial: Macrogol/PEG 3350 plus electrolytes for treatment of patients with constipation associated with irritable bowel syndrome. Am J Gastroenterol. 2013;108(9):1508-15.

28. Lee-Robichaud H, Thomas K, Morgan J, Nelson RL. Lactulose versus Polyethylene Glycol for Chronic Constipation. Cochrane Database Syst Rev. 2010;(7).

29. Di Palma JA, Cleveland MV, Mcgowan J, Herrera JL. An open-label study of chronic polyethylene glycol laxative use in chronic constipation. Aliment Pharmacol Ther. 2007;25(6):703-8.

30. Ahsan MK, Tchernychev B, Kessler MM, Solinga RM, Arthur D, Linde CI, et al. Linaclotide activates guanylate cyclase-C/cGMP/ protein kinase-II-dependent trafficking of CFTR in the intestine. Physiol Rep. 2017;5(11).

31. Eutamene H, Bradesi S, Larauche M, Theodorou V, Beaufrand C, Ohning G, et al. Guanylate cyclase C-mediated antinociceptive effects of linaclotide in rodent models of visceral pain. Neurogastroenterol Motil. 2010;22(3).

32. Videlock EJ, Cheng V, Cremonini F. Effects of Linaclotide in Patients With Irritable Bowel Syndrome With Constipation or Chronic Constipation: A Meta-analysis. Clinical Gastroenterology and Hepatology. W.B. Saunders. 2013;11:1084-92.e3.

33. Atluri DK, Chandar AK, Bharucha AE, Falck-Ytter Y. Effect of linaclotide in irritable bowel syndrome with constipation (IBS-C): A systematic review and meta-analysis. Neurogastroenterol Motil. 2014;26(4):499-509.
34. Rey E, Mearin F, Alcedo J, Ciriza C, Delgado-Aros S, Freitas T, et al. Optimizing the Use of Linaclotide in Patients with Constipation-Predominant Irritable Bowel Syndrome: An Expert Consensus Report. Vol. 34, Advances in Therapy. Springer Healthcare. 2017;34:587-98.

35. Lever E, Cole J, Scott SM, Emery PW, Whelan K. Systematic review: The effect of prunes on gastrointestinal function. Alimentary Pharmacology and Therapeutics. Blackwell Publishing Ltd. 2014; $40: 750-8$.

36. Bijkerk CJ, Muris JWM, Knottnerus JA, Hoes AW, De Wit NJ. Systematic review: The role of different types of fibre in the treatment of irritable bowel syndrome. Vol. 19, Alimentary Pharmacology and Therapeutics. Aliment Pharmacol Ther. 2004; 19:245-51.

37. Suares NC, Ford AC. Systematic review: The effects of fibre in the management of chronic idiopathic constipation. Alimentary Pharmacology and Therapeutics. Aliment Pharmacol Ther. 2011;33:895-901.

38. Mueller-Lissner S, Kamm MA, Wald A, Hinkel U, Koehler U, Richter E, et al. Multicenter, 4-week, double-blind, randomized, placebo-controlled trial of sodium picosulfate in patients with chronic constipation. Am J Gastroenterol. 2010;105(4):897-903.

39. Ford AC, Moayyedi P, Lacy BE, Lembo AJ, Saito YA, Schiller LR, et al. American College of Gastroenterology monograph on the management of irritable bowel syndrome and chronic idiopathic constipation. Am J Gastroenterol. 2014;109(Suppl. 1).

40. Campitelli EH, del V Sivanto M. Uso del prucalopride en el estreñimiento crónico inducido por el uso de opioides en una población ambulatoria. Acta Gastroenterológica Latinoam. 2018;48(4):296-301.

41. Schumann D, Anheyer D, Lauche R, Dobos G, Langhorst J, Cramer H. Effect of Yoga in the Therapy of Irritable Bowel Syndrome: A Systematic Review. Clinical Gastroenterology and Hepatology. W.B. Saunders. 2016;14:1720-31.

42. Aucoin M, Lalonde-Parsi MJ, Cooley K. Mindfulness-based therapies in the treatment of functional gastrointestinal disorders: A Meta-analysis. Evidence-based Complementary and Alternative Medicine. Hindawi Limited. 2014;2014.

43. Ford AC, Quigley EMM, Lacy BE, Lembo AJ, Saito YA, Schiller LR, et al. Effect of antidepressants and psychological therapies, including hypnotherapy, in irritable bowel syndrome: systematic review and meta-analysis. The American journal of gastroenterology. Am J Gastroenterol. 2014;109.

44. Soncini M, Stasi C, Usai Satta P, Milazzo G, Bianco M, Leandro G, et al. IBS clinical management in Italy: The AIGO survey. Dig Liver Dis. 2019;51(6):782-9. 